\title{
Visit the EFSUMB Ultrasound Learning Area at UEG
}

Visit the EFSUMB Ultrasound Learning Area (close to the Endoscopic area) at UEG week for hands-on training, basic and advanced courses in ultrasound for the gastroenterologist and lectures covering liver, GI and the pancreas from expert gastroenterologists.

\section{ueg $w e e k$}

October 15-19, 2016

Venue: Austria Center Vienna

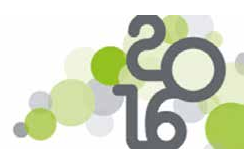

